\title{
EL DESARROLLO INSTITUCIONAL DEL PERSONAL ADMINISTRATIVO DEL CONGRESO NACIONAL EN EL CHILE POST-AUTORITARIO: ¿SUPERPOSICIÓN INSTITUCIONAL O TRAYECTORIA DEPENDIENTE?*
}

\section{THE INSTITUTIONAL DEVELOPMENT OF THE CONGRESSIONAL PERSONNEL IN POST-AUTHORITARIAN CHILE: ¿INSTITUTIONAL LAYERING OR DEPENDENT PATH?}

\section{IVÁN MAURICIO OBANDO CAMINO**}

RESUMEN: Este artículo describe el desarrollo institucional del personal administrativo del Congreso Nacional entre 1990 y 2015, para determinar si constituye un caso de trayectoria dependiente o de superposición institucional. Esta investigación se inclina en el primer sentido, lo que explica la restauración y actualización del modelo organizacional de oficinistas y escribientes legislativos, empleado históricamente para su organización, en dicho periodo.

Palabras clave: Chile, personal administrativo, institución política.

Aвstract: This article describes the institutional development of the administrative personnel of the National Congress from 1990 through 2015 to determine whether such phenomenon constitute a case of dependent path or institutional layering. This research supports the former, which accounts for the restoration and updating of the organizational model of legislative officers and clerks that underlays its historical organization during that period.

KeYwords: Chile; administrative personnel; political institution.

Recibido: 20.02.2020. Aceptado: 25.09.2020.

\section{INTRODUCCIÓN ${ }^{1}$}

T As instituciones se conciben como "las reglas del juego en una so_ciedad" (North, 1990, p. 3), pues determinan la interacción social, influyen en las expectativas de los actores políticos (en adelante los actores)

\footnotetext{
* Agradezco a los dos evaluadores anónimos por sus observaciones y a CONICYT por financiar esta investigación (Proyecto Fondecyt regular $\mathrm{N}^{\circ} 1180581$ ).

${ }^{* *}$ Ph.D en Ciencia Política. Académico de la Universidad de Talca, Talca, Chile. Correo electrónico: iobandoc@utalca.cl. Orcid: https://orcid.org/0000-0002-3307-9400

${ }^{1}$ Abreviaturas: Ent.: entrevista; LOCCN: Ley Orgánica Constitucional del Congreso Nacional; Ley: L.
} 
y proyectan poder hacia el futuro (North, 1990, p. 3; Pierson, 2015, pp. 128-130; Rosenblatt y Toro, 2015, pp. 256-261; Capoccia, 2016, pp. 9-13).

Dada la ubicuidad de las instituciones, este trabajo describe el desarrollo de una institución, que es el personal administrativo del Congreso Nacional (en adelante el personal), en el Chile post-autoritario entre 1990 y 2015, para determinar si aquel desarrollo constituye un caso de trayectoria dependiente o de superposición institucional, a la luz de los aportes del institucionalismo histórico de la ciencia política (Pierson, 2004, pp. 17-48; Mahoney y Thelen, 2010, pp. 14-22; Mahoney et al., 2016, pp. 82-86).

El personal constituye una burocracia de carrera, jerarquizada, neutral y profesional, dirigida por funcionarios elegidos por la mayoría parlamentaria y constituida por los que laboran en los distintos servicios del Congreso Nacional, i.e. Cámara de Diputados (en adelante la Cámara), Senado y Biblioteca del Congreso Nacional (en adelante la Biblioteca), conforme a un vínculo jurídico estatutario, con exclusión de parlamentarios y asesores personales. Este personal colaboró en la superación de problemas derivados de la reapertura del Congreso Nacional, permitiendo a este último adquirir capacidades técnicas (Palanza et al., 2013, p. 29).

El personal fue organizado bajo un modelo de oficinistas y escribientes legislativos que circunscribió sus labores a transcribir debates, redactar informes y administrar, y que se consolidó mediante la creación de oficinas y escalafones desde fines del siglo XIX. Aproximadamente un tercio de sus integrantes fue desvinculado tras la clausura del Congreso Nacional en 1973 y los supervivientes fueron destinados en comisión de servicios a distintos órganos, incluida la Junta de Gobierno (en adelante la Junta), la que proveyó solo algunas vacantes y agregó a los escalafones del personal otros de carácter administrativo ${ }^{2}$. Esta última dictó la Ley $\mathrm{N}^{\circ} 18.918$, en adelante la LOCCN, a principios de 1990, la que dispuso que una ley fijaría las plantas y rentas del personal, brindó inamovilidad a este y estableció que los reglamentos internos regularían, en lo demás, su estatuto, aplicándose supletoriamente las normas de la Administración Pública. Con todo, la inexistencia de un parlamento bajo el autoritarismo debilitó al personal, el que contaba con una misión y planta en extinción hacia $1990^{3}$, lo que llevó a la contratación de funcionarios después de marzo de 1990. Posteriormente, el Congreso Nacional confirió al personal un régimen estatu-

\footnotetext{
${ }^{2}$ Hacia 1989 se habían agregado tres escalafones en la Cámara, dos en el Senado y substituido el único escalafón de la Biblioteca.

${ }^{3}$ La dotación máxima de funcionarios disminuyó de 478 en 1972 a 375 en 1981, según la ley de presupuestos.
} 
tario especial mediante la L. $\mathrm{N}^{\circ} 19.297$ y su Acuerdo Complementario de 1994, creándose el Consejo Resolutivo de Asignaciones Parlamentarias y el Comité de Auditoría Parlamentaria, como órganos comunes, mediante la L. $\mathrm{N}^{\circ} 20.447$ de 2010.

En consecuencia, la pregunta planteada supra obedece a la persistencia aparente de las estructuras organizativas del personal creadas en 1994, por lo que existe la interrogante de si el modelo organizacional de oficinistas y escribientes legislativos, presente en sus orígenes, evolucionó posteriormente hacia algún modelo diverso.

Respecto de la metodología, esta es una investigación descriptiva consistente en un estudio de caso disciplinado-configurativo, el que describe las circunstancias que incidieron en el desarrollo institucional del personal, para lo cual el marco teórico permite guiar sistemáticamente la investigación e interpretar teóricamente los hallazgos (Eckstein, 1993).

Dicho marco considera las opciones de trayectoria dependiente y superposición institucional, ya que discurren sobre la mantención formal de las reglas preexistentes. Ellas han servido para analizar políticas y programas (Streeck \& Thelen, 2005; Mahoney \& Thelen, 2010; Sorensen, 2015; Aagard, 2017); reformas económicas (North, 1990; Caballero, 2008; Thelen, 2019), regímenes políticos (Mahoney, 2001; Baeza, 2012; Chagas, 2016) y comisiones y normas parlamentarias (Schickler, 2001, 2005; Hoffmann \& Loëwenberg, 2009), siendo este uno de los primeros estudios que las emplea para analizar el desarrollo institucional del personal administrativo de una organización política, acorde a compilaciones efectuadas por autores (Thelen \& Mahoney, 2015, pp. 28-31).

Asimismo, es un estudio de caso diacrónico sobre el desarrollo institucional del personal, el que fue delimitado para incluir la reapertura del Congreso Nacional, la creación del nuevo régimen estatutario del personal y el impacto de los órganos comunes creados en 2010. La extensión del periodo y hallazgos reportados para periodos previos (Tapia, 1960, pp. 32-34; Obando, 2011, pp. 187-2013; Obando, 2016, pp. 125-152), permiten descartar un sesgo de selección del caso, habiéndose rastreado "los arreglos institucionales a través del tiempo" (Pierson, 2004, p. 140) para contrastar los nuevos hallazgos con el desarrollo histórico del personal.

Igualmente, es una investigación cualitativa para cuya ejecución se administraron entrevistas semi-estructuradas a una decena de personas. Ellas fueron tres exparlamentarios y tres parlamentarios elegidos en 1990, quienes se desempeñaron en una o ambas cámaras durante todo o parte del periodo, cuatro de los cuales integraron las Mesas y comisiones de régimen 
interno; un exfuncionario que desempeñó un cargo de jefatura en una cámara durante la mayor parte del periodo; un funcionario directivo de una de las cámaras incorporado tras 1990; y dos académicos que interactuaron con el personal tras $1990^{4}$. Finalmente, se analizaron diarios de sesiones, recopilaciones legales e informes parlamentarios, publicados entre 1990 y 2010, disponibles en bibliotecas nacionales.

En síntesis, este trabajo sostiene que este es un caso de trayectoria dependiente, en el que un conjunto de condiciones propició la restauración y actualización del modelo organizacional tradicional del personal en 1994, sin que cambios puntuales posteriores comportaren modificaciones sistémicas al mismo y al funcionamiento del personal.

Finalmente, este trabajo consta de tres secciones, las que discurren sobre el marco teórico, los hallazgos de la investigación, y su análisis y discusión.

\section{MARCO TEÓRICO}

\subsection{La trayectoria dependiente y el equilibrio puntuado o interrumpido}

La persistencia de instituciones sugiere su continuidad, estabilidad e inercia y su funcionamiento genera retroalimentación positiva y recursos de poder -incluso culturales- para quienes las apoyan (Thelen, 2009, pp. 474475; Galvin, 2012, pp. 2-3, Pierson, 2015, pp. 133-134; Capoccia, 2016, pp. 22-23; Busemeyer \& Thelen, 2020, p. 27).

Estas ideas fueron recogidas en la teoría de la trayectoria dependiente, según la cual las elecciones iniciales y contingentes pueden tener impactos de largo plazo, generando caminos institucionales difíciles de modificar, aunque dichas elecciones hubieren sido subóptimas (Sorensen, 2015, pp. 21-22; Fioretos et al., 2016, pp. 10-12; Conran \& Thelen, 2016, pp. 54-56; Mahoney et al., 2016, pp. 82-86; Bosch, 2018, p. 22). No obstante, North (1990) precisó que dicha trayectoria solo implica que la "historia importa" (p. 100), sin ser inevitable, y constituye "una forma de estrechar conceptualmente el conjunto de opciones y vincular la toma de decisiones a través del tiempo" (p. 98).

La trayectoria dependiente puede ser concebida en términos amplios o precisos. Sewell sostuvo lo primero al decir que "lo que ha sucedido más tempranamente en el tiempo afectará los resultados posibles de una se-

\footnotetext{
${ }^{4}$ Diez de veintiún personas accedieron a ser entrevistadas y su identidad es confidencial.
} 
cuencia de eventos que tenga lugar más adelante" (Pierson, 2004, p. 20); mientras que Levi (1997) representó lo segundo al decir que "una vez que un país o una región ha comenzado a transitar por una pista determinada, los costos de una reversión son muy altos" (p. 28), lo que sugiere una secuencia auto-reforzante de eventos constituida por "procesos sociales que exhiben retroalimentación positiva y generan patrones de ramificación de desarrollo histórico" (Pierson, 2004, p. 21).

El análisis económico advirtió que retornos crecientes de escala se producen por efectos de coordinación y aprendizaje, adaptación de expectativas y costos fijos, permitiendo la predominancia de arreglos subóptimos por eventos contingentes, como consecuencia de múltiples equilibrios, posibles ineficiencias, cierre de soluciones y trayectoria dependiente (North, 1990, pp. 93-94; Sorensen, 2015, p. 21). La percepción de tales retornos permite aplicar la trayectoria dependiente al análisis del desarrollo institucional, pues la adopción de ciertas instituciones relega a segundo plano otras alternativas viables y genera retroalimentación positiva para quienes las apoyan y cambio incremental en las mismas (North, 1990, pp. 95, 99, 103-104, 112; Thelen, 2003, pp. 220-221; Sorensen, 2015, p. 21).

Pierson (2004) sostuvo que la retroalimentación positiva es predominante en política por la centralidad de la acción colectiva, su alta densidad institucional, el realce de asimetrías de poder mediante la autoridad política y su complejidad y opacidad intrínseca. Él señaló que dicha intensidad obedece a la ineficacia de mecanismos compensatorios presentes en la economía, dada la ineficiencia de la competición y el aprendizaje, los horizontes de corto plazo de los actores y el sesgo pro statu quo de las instituciones (Pierson, 2004, pp. 31-40)5. Por ello Campbell (2011) y Lindegaard (2013) dijeron que las instituciones suelen generar trayectorias dependientes, ya que tienen altos costos iniciales, son difíciles de modificar por diseño, los actores son renuentes al cambio por el conocimiento acumulado, sus beneficiarios apoyan su continuación, su permanencia limita el rango de lo posible o apropiado para los actores y el entrelazamiento de instituciones hace difícil cambiar alguna aisladamente.

Esta teoría inspiró un modelo de cambio institucional, denominado de equilibrio puntuado o interrumpido, para el cual la creación institucional sucede en momentos históricos de elección, denominadas coyunturas críticas, sin que la elección inicial pueda dejarse sin efecto (Krasner, 1984, pp.

\footnotetext{
${ }^{5}$ Mahoney et al. (2016, pp. 84-86) identificaron una corriente -i.e. Posner- basada en una secuencia reactiva de eventos que conduce al resultado.
} 
240-244). Estas últimas consisten en episodios de interrupción abrupta de periodos de estabilidad institucional, dando lugar a una crisis de legitimidad, mayor incertidumbre y cambio significativo, seguido de un periodo de estabilidad institucional en que un nuevo poder se proyecta al sistema político (Thelen, 2009, p. 474; Capoccia, 2015, pp. 158-160; Pierson, 2015, pp. 132-134; Bosch, 2018, p. 22). En este modelo factores exógenos (e.g. revolución) desequilibran los arreglos institucionales, pero su naturaleza "dependerá de la institución y el contexto" (Sorensen, 2015, p. 25), y la estructura constreñirá menos a los actores (Conran \& Thelen, 2016, p. 55). En estas circunstancias, la contingencia representa "(...) la apertura de múltiples futuros posibles, la determinación del cual dependerá de la dinámica política particular y de las relaciones de poder (...)" (Sorensen, 2015, p. 25).

Frente a ello, Campbell (2011) propuso una aproximación metodológica -pragmática- para comprender el cambio no abrupto en instituciones dependientes de trayectoria, distinguiendo dos procesos de mezcla institucional: bricolaje y traducción. El bricolaje es "el reordenamiento y recombinación de principios y prácticas institucionales en formas nuevas y creativas" (Campbell, 2011, p. 98), mientras que la traducción es la "mezcla de nuevos elementos en arreglos institucionales preexistentes" (Campbell, 2011, pp. 98-99), lo que supone el ajuste de los primeros a los segundos.

\subsection{El cambio institucional gradual y la superposición institucional}

Mahoney \& Thelen (2010) observaron que las instituciones distribuyen poder entre coaliciones de actores, siendo relevantes el apoyo y los equilibrios de fuerzas, por lo que "el cambio y la estabilidad están en el hecho inextricablemente unidos" (Mahoney \& Thelen, 2010, p. 9); en consecuencia, los procesos de reproducción y cambio institucional requieren concentrarse en coaliciones de actores que brindan apoyo a las instituciones (Thelen, 2014, pp. 206-207; Bosch, 2018, p. 22; Thelen, 2019, pp. 300, 312). Por lo mismo, al interior de las instituciones se generan presiones para el cambio, sin que pueda presumirse la conformidad de los actores con las reglas (Mahoney \& Thelen, 2010, pp. 10-11).

$\mathrm{Al}$ respecto, las instituciones experimentan cambios graduales y frecuentes inducidos por factores endógenos, los que tienen efectos acumulativos de largo plazo, debiendo considerarse las características del contexto político y de la institución, pues ellas determinan tipos modales de cambio institucional gradual, uno de los cuales es la superposición institucional (Thelen, 2003, pp. 292-293; Thelen, 2004, pp. 209-211, 226-228; Mahoney \& Thelen, 2010, pp. 16-17; Aagard, 2017, p. 281). 
Esta última consiste en agregar nuevas reglas institucionales a las existentes, mediante enmiendas, adiciones o revisiones que alteran el ordenamiento del comportamiento social por las segundas, pero sin reemplazarlas formalmente (Mahoney y Thelen, 2010, pp. 16-17; Aagard, 2017, p. 281). La superposición institucional modifica una trayectoria institucional mediante un mecanismo de crecimiento diferencial que disminuye el impacto de las instituciones existentes y atrae apoyo hacia nuevas instituciones (Streeck \& Thelen, 2005, p. 23; Aagard, 2017, p. 282).

La superposición institucional supone que los defensores del status quo institucional tienen fuerte capacidad de veto y que existe bajo nivel de discreción en la interpretación de las reglas institucionales (Mahoney \& Thelen, 2010, pp. 18-19); asimismo, esta tiene un agente de cambio -subversivo- que persigue cambiar el statu quo institucional sin revelar sus objetivos, mediante estrategias de corto plazo (Mahoney \& Thelen, 2010, pp. 25-26). Por último, la superposición institucional implica la unión de ideas de equilibrio puntuado y cambio incremental, dado su enfoque en actores e instrumentos (Van der Heijen, 2011, p. 14-16).

\section{HALLAZGOS DE LA INVESTIGACIÓN}

\subsection{Primeros años de funcionamiento del Congreso Nacional}

El Congreso Nacional reinició sus actividades con el personal preexistente, además de contrataciones puntuales efectuadas antes del 11 de marzo de 1990. El funcionamiento en Valparaíso planteó problemas administrativos y acentuó la insuficiencia del personal, procediéndose a la contratación apresurada de funcionarios; de hecho, una subcomisión de la Comisión de Régimen Interno de la Cámara evaluó currículums vitae propuestos por diputados (Biblioteca del Congreso Nacional, s.f., pp. 15, 35, 42, 47, 53; Ent. 18/01/2019, 29/01/2019, 27/02/2019, 18/08/2019). La capacitación de estos funcionarios correspondió a los de planta preexistentes.

Los funcionarios se organizaron y presionaron gradualmente sus demandas sobre las Mesas, las que consistían en estabilidad funcionaria y mejora remuneratoria (Ent. 22/01/2018, 18/08/2019, 30/01/2019). Lo primero suponía mantener la inamovilidad funcionaria y crear una planta con cargos diversos (Ent. 04/03/2019) y diferenciados por escalafones en la Cámara (Ent. 18/01/2019). Los funcionarios antiguos deseaban mantener su estabilidad laboral, mientras los nuevos deseaban su incorporación a la planta en condiciones similares; con todo, el empleo de los primeros 
no estuvo amenazado, porque la conformación de las Mesas (UDI-Concertación) propició decisiones consensuadas sobre el personal, y los funcionarios influyeron en el corto plazo en los parlamentarios, sin perjuicio de tener redes en los partidos (Ent. 22/01/2019, 29/01/2019, 27/02/2019, 04/03/2019, 05/03/2019, 18/08/2019). Lo segundo suponía contar con una escala de remuneraciones con emolumentos equivalentes a los de cargos similares en el sector público y privado (Ent. 04/03/2019).

Dada la inexistencia de la ley sobre planta y rentas del personal, este podía ser organizado hipotéticamente de maneras diversas. Una posibilidad era restaurar y actualizar el modelo organizacional tradicional y reconstituir el personal como profesional, jerarquizado y neutral, lo que satisfacía sus demandas, reducía la desconfianza parlamentaria y resguardaba la influencia partidista, y tenía antecedentes en Chile y Europa. También se podía organizar el personal siguiendo un modelo de expertos en legislación y política pública, nombrados por la mayoría parlamentaria y a cargo del análisis en comisiones, sin perjuicio del personal para labores de apoyo (e.g. Estados Unidos), lo que incrementaba la influencia parlamentaria y disminuía la presión por crear una planta y la influencia partidista. Finalmente, los expertos podían constituir un escalafón profesional de la planta, basándose en la Secretaría de Coordinación Legislativa y de Asesoría Técnico Profesional prevista en la LOCCN (República de Chile, 1989, pp. 4948), lo que originaba problemas de coordinación, generaba desconfianza sobre la neutralidad de los expertos y afectaba la influencia partidista.

Las demandas persuadieron a la Cámara de contratar a Price Waterhouse para hacer un diagnóstico sobre la organización, funciones y remuneraciones con en 1991, lo que el personal no respaldó (Ent. 18/08/2019), pero sus recomendaciones fueron rechazadas por su origen externo (Ent. $22 / 01 / 2019,18 / 08 / 2019)$ y por carecer de una visión sistémica sobre cada servicio (Ampuero, 2012, p. 121). El Senado hizo algo similar con otra consultora (Cámara de Diputados, 1994, pp. 3807, 3846). Con todo, la situación del personal no figuró entre las prioridades de muchos parlamentarios (Ent. 22/01/2019, 30/01/2019, 27/02/2019, 05/03/2019, 18/08/2019), por lo que las Mesas y comités terminaron por actualizar lo existente ${ }^{6}$, regularizar la planta para acoger las demandas del personal ${ }^{7}$ y crear nuevos car-

\footnotetext{
${ }^{6}$ Un Diputado señaló que "[s]e recogió la experiencia de un Congreso Nacional que funcionó por décadas implícitamente" (Ent. 05/03/2019) y un Senador sostuvo que "[e]ra simplemente reaccionar frente a las exigencias que se tenían" (Ent. 27/02/2019).

${ }^{7}$ Un ex-Diputado expresó: "Algo característico del Parlamento en esta época es que las plantas se fueron ampliando para no generar conflictos entre los funcionarios, y la práctica de régimen interno fue recepcionar las demandas y pedir los recursos al gobierno, sin mayor reflexión" (Ent. 30/01/2019).
} 
gos sin competencias definidas (Oficina de Informaciones de la Cámara de Diputados, 2009, 81-82; Ampuero, 2012, pp. 123-124; Ent. 18/01/2019, 22/01/2019, 29/01/2019, 30/01/2019, 27/02/2019, 04/03/2019, 05/03/2019, $18 / 08 / 2019)$.

\subsection{La creación del régimen estatutario del personal}

En 1994 se dictó la L. N 19.297, modificatoria de la LOCCN, basada parcialmente en propuestas de funcionarios y sus asociaciones. El tiempo transcurrido obedeció al desinterés de los parlamentarios y la presión de terceros, incluidos funcionarios y parlamentarios (Biblioteca del Congreso Nacional, s.f., pp. pp. 46-46, 131)

Esta ley dispuso que el régimen estatutario del personal fuera regulado por reglamentos internos de cada servicio y que se aplicaran supletoriamente las normas de la Administración Pública. Además, estableció que el acceso a los cargos sería por concurso público y que la planta se distribuiría en diecisiete categorías en la Cámara, el Senado y la Biblioteca, y existiría un número fijo de cargos por categoría y un total de 629 cargos; es decir, la ley contempló un esquema de plantas e inmovilizó el número de cargos por categoría, limitando las presiones de los funcionarios?.

La ley dilucidó que la administración del personal y unidades de cada servicio correspondería al Secretario respectivo y al Director de la Bibliote$\mathrm{ca}$, como Jefe Superior del Servicio, sin perjuicio de la supervigilancia administrativa de la comisión respectiva. Igualmente, la mayoría de las unidades previstas en la ley tenían antecedentes en la historia parlamentaria (e.g. Secretarías, Biblioteca), salvo un Centro de Informática y Computación.

La ley dispuso que una comisión bicameral crearía, por una sola vez, los escalafones, escalas de remuneraciones y beneficios remuneratorios, entre

\footnotetext{
${ }^{8}$ Según un ex-Diputado: "En relación con la demora en la tramitación de la ley de 1994, los funcionarios ponían trabas y había miles de preocupaciones, por lo que el tema del personal era secundario" (Ent. 18/8/2019). Otro ex-Diputado sostuvo: “(..) los funcionarios se vinculan a dos o tres parlamentarios. (...) en cada bancada hay dos o tres parlamentarios con hipersensibilidad respecto de los funcionarios" (Ent. 27/02/2019).

${ }^{9}$ Un ex-Diputado afirmó: "Se había estirado demasiado el chicle en cuanto a la extensión de la planta del personal, porque el gobierno iba a decir que no aumentaba, produciéndose comparaciones con el Senado. Se equiparaban y se asimilaban en carácter ascendente en cuanto a recursos y personal" (Ent. 30/01/2019). Otro ex-Diputado señaló: “También se modificó la ley del Congreso Nacional para evitar la costumbre que los funcionarios pedían beneficios separadamente, lo que generaba una escalera sin fin y la ley estableció las bases de la planta, para que las Comisiones de Régimen Interior no se vieran presionadas cada cierto tiempo. (...) Las Comisiones de Régimen Interno tienden a tener manga ancha, lo que generaba una gestión inorgánica. Por lo anterior, no querían escalas esquemáticas en 1994" (Ent. $18 / 08 / 2019$ ).
} 
otros, dentro de 180 días siguientes a su publicación. Luego, cada Jefe Superior encasillaría al personal de planta, en funciones al 31 de diciembre de 1993, sin solución de continuidad, en los escalafones y plantas con igual denominación y en el mismo cargo; en caso contrario, el encasillamiento sería en un cargo de grado equivalente. Los empleos vacantes serían provistos por cada Jefe Superior, cumpliendo los requisitos reglamentarios, mediante el ascenso del personal de planta, el nombramiento del personal a contrata o a honorarios en funciones a esa fecha previo concurso interno, o mediante concurso público. La ley exceptuó de dichos requisitos a quienes, a la misma fecha, estaban a contrata u honorarios, pero que hubieran pertenecido a la planta y hubieran sido exonerados por motivos políticos, previa evaluación del Jefe Superior, a propuesta de la comisión respectiva. Así, el concurso público para el ingreso a la planta no fue exigido con alcance general.

La comisión bicameral procuró resguardar el profesionalismo y establecer rentas compatibles con otros sectores e igualdad salarial entre quienes desempeñaban funciones y responsabilidades semejantes. Esta comisión propuso un Acuerdo Complementario que estableció categorías de renta para distintos niveles de funcionarios; beneficios remuneratorios (algunos superiores a los de otros empleados públicos por una defensa corporativa del personal) (Ent. 30/01/2019, 18/08/2019); requisitos profesionales y/o de experiencia para algunos cargos, y los escalafones del personal, contemplando diez para cada cámara (con denominaciones idénticas) y once para la Biblioteca. Esta propuesta fue aprobada a fines de agosto de 1994, pero las asociaciones de funcionarios manifestaron desconocer su contenido final (Cámara de Diputados, 1994, pp. 3810-3811, 3849-3859; Senado, 1994), tras lo cual cada servicio dictó su reglamento interno del personal.

Varios escalafones fueron continuadores de estructuras organizativas y/o funciones previas de las cámaras (e.g. Secretaría, Redacción de Sesiones, Oficina de Informaciones, Relaciones Públicas), mientras otros (e.g. Cargos Complementarios, Profesional y Técnico de Administración, Servicios) incorporaron funciones relativas al ambiente parlamentario (e.g. Contraloría, Mantención, Informática, Administración, Abastecimiento y Logística, Coordinación y Supervisión de Servicios). La Biblioteca aumentó en complejidad por su funcionamiento como biblioteca parlamentaria y cambios organizacionales provenientes de su proyecto de modernización (Feliú, 2004, pp. 66-68).

En definitiva, la L. $\mathrm{N}^{\circ} 19.297$ y su Acuerdo Complementario se tradujeron en una restauración y actualización del modelo organizacional tradicional del personal; de hecho, en la Cámara "la planta mantuvo, en gran 
medida, la antigua organización interna y sancionó numerosas situaciones de hecho" (Oficina de Informaciones de la Cámara de Diputados, 2009, p. 72$)^{10}$. Por ello las iniciativas de modernización, e.g. el Proyecto de Modernización Parlamentaria, incorporaron tecnologías de información y resolvieron problemas competenciales, pero no modificaron el modelo organizacional y funcionamiento del personal (Ent. 04/12/2018, 18/01/2019, 29/01/2019).

\subsection{La implementación del régimen estatutario del personal}

El nuevo régimen estatutario no se tradujo en la provisión de todos los cargos de planta por desacuerdo en las comisiones respectivas, generando un congelamiento de la planta (Ent. 18/01/2019, 18/08/2019). Además, la evaluación de sus efectos fue crítica, porque sus reglas institucionales favorecieron rutinas y estructuras disfuncionales y resistentes al cambio (Ent. 18/01/2019, 29/01/2019).

Respecto de la Cámara, su Oficina de Informaciones detectó interferencia parlamentaria en administración; indeterminación de competencias; defensa corporativa de escalafones, servicios, oficinas y su autonomía; inexistencia de un organigrama (aprobado en 2000); crecimiento inorgánico de la planta; y una descripción de funciones inexistente, entre otros (Oficina de Informaciones de la Cámara de Diputados, 1996, pp. 3-7). En parte, esto obedeció a que aquella "adoptó como estructura organizacional y de funcionamiento aquella que tenía (...) antes de 1973, por lo que, a poco andar, se fueron adaptando, de hecho, las distintas estructuras y ámbitos competenciales de cada estamento. (...)" (Cámara de Diputados, 1996, p. 296). Igualmente, Brahm (1997) observó que la rigidez estructural de la planta dificultaba su adaptación al cambio, lo que vinculó al liderazgo parlamentario, ya que "(...) influyen en esta inercia los estamentos directivos, dada la naturaleza de la generación de sus cargos, la rotación a que se someten y la dispersión del poder que es propia de los parlamentos" (p. 26).

La creación de subsecretarías en 2000 buscó ejecutar propuestas derivadas de estos diagnósticos, confiriéndose la gestión de escalafones a una Subsecretaría Administrativa, sin obtenerse el propósito en el corto plazo (Oficina de Informaciones de la Cámara de Diputados, 2009, p. 81; Ampuero, 2012, p. 122). Para introducir mejoras en la gestión, la Mesa contrató a

\footnotetext{
${ }^{10}$ Un ex-Senador indicó: "Yo creo que la ley de 1994 normaliza, dentro de las dificultades propias de un organismo en transición y no se pretendió darle un vuelco tal que nos diera una estructura del personal del Senado" (Ent. 22/01/2019).
} 
Procesac, la que reportó inexistencia de descripción de funciones e incongruencia del desempeño respecto de la organización (Ampuero, 2012, p. 123). Esto reintrodujo la modernización parlamentaria, sumándose problemas de promoción, transparencia, automatización y gestión de nuevas funciones, lo que fue objeto de la ejecución de nuevos acuerdos y proyectos (e.g. Proyecto PNUD sobre Fortalecimiento de la Ética y Transparencia en el Congreso Nacional) (Ampuero, 2012, pp. 123-124; Gobierno de Chile, s.f., pp. 5-7). De ahí que su Oficina de Informaciones propusiera la reconstrucción organizativa de aquella para no repetir errores, pues su organización "es el resultado de la estructura que fue tradicional hasta 1973, más la suma de organismos que, por diversas circunstancias, lograron asumir una cierta especialización e identidad profesional" (Oficina de Informaciones de la Cámara de Diputados, 2009, p. 89).

En cuanto al Senado, los fenómenos descritos se deslizan en comentarios como los de un Senador, para quien

[a] partir de la ley orgánica constitucional y el reglamento histórico de la cámara, se considera que la inercia es la que manda. (...) Hay algunas propuestas de cambio, pero es tal la inercia institucional y el poder de los funcionarios, que es muy difícil. (...) No hay un proyecto institucional en su conjunto y los cambios son reactivos. Se sobreponen y no alteran el funcionamiento. (...) Hay resistencia institucional. (...). (Ent. 27/02/2019)

Otro Senador señaló: "En cuanto a la estructura y funcionamiento del personal, creo que se advierte una continuidad en ambos (...)" (Ent. 04/03/2019). Finalmente, un ex-Senador sostuvo: “(...) se modernizó el parlamento con nuevos parámetros, (...) Sin embargo, (...) las rutinas no cambiaron" (Ent. 18/08/2019).

En cuanto a la Biblioteca, esta se consolidó y expandió tras su Proyecto de Modernización (Ent. 22/01/2019, 18/08/2019). Posteriormente, se ejecutaron proyectos adicionales (e.g. Sistema de Administración y Finanzas, Sistema de Archivos de Prensa, Mejoramiento Red de Comunicaciones del Congreso) y se crearon unidades de apoyo legislativo (e.g. Estudios y $\mathrm{Pu}-$ blicaciones, Apoyo al Proceso Legislativo, Asistente Legislativo, Servicio de Asesoría Parlamentaria, entre otros) (Feliú, 2004, p. 69; Soto, 2015, p. 48). Esto no la dejó exenta de críticas, como las de un Senador, para quien "[1] os órganos creados en 2010 muy poco han impactado en el funcionamiento del Congreso. Lo mismo se puede decir de la Biblioteca del Congreso Nacional, en que algunas modificaciones no fueron razonables" (Ent. 
04/03/2019), mientras que otro Senador sostuvo que "la Biblioteca vive su propia vida y está aún en proceso su colaboración con el procedimiento legislativo, lo que fue muy resistido por ella. (...)" (Ent. 27/02/2019).

El Centro de Informática y Computación nunca fue creado y cada servicio estableció su unidad o escalafón después de 1994. Los órganos comunes fundados en 2010 carecieron de impacto -siquiera numérico- en el desarrollo institucional y funcionamiento del personal (Ent. 18/01/2019, $30 / 01 / 2019,04 / 03 / 2019)$, porque solo persiguieron resguardar la probidad pública (Ent. 22/01/2019).

La duración anual de las Mesas desde mediados de los 90 limitó la autoridad de sus presidentes para innovar institucionalmente, lo que llevó a un Senador a sostener: "Las Mesas no tienen capacidad de levantar una gestión. Se intenta que el Secretario sea el Gerente y Régimen Interno no tiene autoridad o poder, como tampoco los Presidentes de las Cámaras. (...)" (Ent. 27/02/2019). Lo mismo se sigue para modificaciones institucionales, como señaló otro Senador: "El Parlamento tiene un problema y este es el corto plazo que duran las Mesas de cada cámara, que es de solo un año (...). Cada vez que se han ido cambiando aspectos institucionales, ha habido un menor seguimiento de cómo funcionan las instituciones" (Ent. 04/03/2019).

\section{ANÁLISIS Y DISCUSIÓN}

La reapertura del Congreso Nacional permitió al personal recobrar relevancia institucional. La inexperiencia y desinterés de los parlamentarios, la existencia de Mesas consensuadas, las exigencias del trabajo parlamentario y la influencia de los funcionarios, especialmente antiguos, favorecieron la restauración y actualización del modelo organizacional tradicional del personal, por lo que las iniciativas de modernización del periodo no implicaron cambios sistémicos para aquel, debido a la inexistencia de acuerdo partidario y la inercia generada.

Este caso no puede ser considerado como una superposición institucional, porque las reglas institucionales aprobadas en 1994 restauraron y actualizaron el modelo precitado, el que subsistía débilmente desde 1973. Pese a las coincidencias, dichas reglas reemplazaron formalmente las preexistentes, en lugar de superponerse a ellas. Tampoco las reglas puntuales aprobadas posteriormente generaron un mecanismo de crecimiento diferencial que modificare aquellas aprobadas en 1994, ni menos alteraron sen- 
siblemente la forma como el modelo organizacional configuró el comportamiento del personal, cuyos papeles provenían de la concepción originaria de aquel.

Por consiguiente, el caso analizado debe ser considerado como un caso de trayectoria dependiente, en el que el contexto político limitó la extensión de la incertidumbre y de la contingencia entre 1990 y 1994. En lugar de generarse una coyuntura crítica, un conjunto de condiciones -referidas supra- propició la restauración y actualización del modelo organizacional antedicho, lo que condujo a las reglas aprobadas en 1994. Estas permitieron reproducir patrones de comportamiento ligados a las funciones del personal y generar una retroalimentación positiva para este último, cuyos integrantes apoyaron el statu quo institucional, pese a los desacuerdos iniciales, la inercia generada y las dificultades de gestión ${ }^{11}$.

Basados en Campbell (2011), se puede sostener que los cambios puntuales introducidos después de 1994 representan un caso de "traducción", habiendo sido incrementales, no abruptos, como tampoco transformativos y supusieron un ajuste a las reglas preexistentes. En consecuencia, la continuidad caracterizó el desarrollo institucional del personal, pese a las modernizaciones del periodo (Ent. 26/11/2018, 04/12/2018, 18/01/2019, 22/01/2019, 27/02/2019, 04/03/2019, 05/03/2019, 18/08/2019).

Esto último fue posible por la resiliencia de las reglas institucionales adoptadas en 1994. Al respecto, Rosenblatt y Toro (2015) propusieron un mecanismo causal explicativo de la resiliencia institucional ${ }^{12}$, el que distingue entre arreglos cooperativos según dos dimensiones: rango de la cooperación y temporalidad del acuerdo cooperativo (Rosenblatt y Toro, 2015, pp. 257, 262-264). La primera dimensión se refiere al carácter incluyente o excluyente del arreglo institucional, debiendo clarificarse que para su inclusividad se requiere que "haya existido la capacidad de involucrar y representar la mayor cantidad posible de perspectivas, más allá de la cantidad de individuos directamente involucrados" (Rosenblatt y Toro, 2015, p. 265), mientras que la segunda dimensión se refiere a la oportunidad del acuerdo respecto del estallido de un conflicto mayor y distingue entre acuerdos preventivos y reactivos (Rosenblatt y Toro, 2015, p. 266). Estas dimensiones permiten visualizar cuatro tipos de acuerdos cooperativos: a) Reactivo-ex-

\footnotetext{
${ }^{11}$ Un Senador expresó: "Yo creo que acá muy pocos se preocupan de la institución. (...) no es su preocupación [de los funcionarios] el mejoramiento institucional. Nunca les he oído que fueran partidarios de un cambio organizacional. (...)" (Ent. 27/02/2019).

${ }^{12} \mathrm{La}$ resiliencia institucional "supone la capacidad de permanecer estable a pesar de la emergencia de conmociones exógenas” (Rosenblatt y Toro, 2015, p. 257).
} 
cluyente: acuerdos adoptados por un líder o grupo para reaccionar ante un conflicto; b) Reactivo-incluyente: acuerdos inclusivos para reaccionar ante un conflicto; c) Preventivo-excluyente: acuerdos adoptados por un líder o grupo para prevenir un conflicto; d) Preventivo-incluyente: acuerdos inclusivos para prevenir un conflicto (Rosenblatt y Toro, 2015, pp. 266-267). Para los autores mencionados la resiliencia institucional es mayor en acuerdos incluyentes, especialmente cuando son preventivos, porque permiten una reproducción del consenso institucional y probablemente un cambio adaptativo y gradual; en cambio, los acuerdos excluyentes, sean o no reactivos, están expuestos a impugnación creciente y tienen menor resiliencia institucional (Rosenblatt y Toro, 2015, pp. 268-269).

De acuerdo a lo expuesto, las reglas institucionales adoptadas en 1994 constituyeron una decisión preventiva-incluyente. Estas reglas consideraron los intereses de los diferentes actores (i.e. estabilidad laboral, remuneración, beneficios y funcionamiento interno), pese a desacuerdos específicos (e.g., plantas esquemáticas de personal), y fueron elaboradas con anticipación al estallido de un conflicto mayor, mediante consultas parciales con el personal realizadas antes y durante el proceso de decisiones, lo que permitió algún grado de influencia al personal. Esta representación de intereses generó un equilibrio institucional, cuya arquitectura fue mantenida en el periodo por el personal y el liderazgo parlamentario, ya que solo podía ser revisado conforme al proceso de elaboración de las leyes o reglamentos parlamentarios, lo que facilitó la reproducción del consenso (Rosenblatt y Toro, 2015, pp. 267-268).

Los hallazgos informados en esta investigación están parcialmente acordes al consenso emergente en el institucionalismo histórico de la última década. Al igual que este último, estos hallazgos descartan el equilibrio puntuado o interrumpido como modelo recurrente de cambio institucional, pero se apartan de aquel al no evidenciar un proceso de cambio institucional distributivo, transformativo y consecuencial, como sería el caso de la superposición institucional, entre otros (Djelic, 2010, p. 33; Fioretos et al., 2016, pp. 12-14).

Esto no implica refutación de las teorías de cambio institucional gradual, sino que sugiere que el desarrollo institucional -y el cambio institucional- debe ser entendido como un proceso que se desenvuelve en el tiempo, pudiendo asumir connotaciones incrementales (e.g., dependencia de trayectoria, bricolaje y traducción) o transformativas (e.g., teorías de cambio gradual) según el contexto, actores y ámbitos del sistema político (Hall, 2011, p. 219; Pierson, 2015, pp. 131-141; Hall, 2016, pp. 38-39), por lo que diversos aspectos de un fenómeno político pueden ser explicados mediante 
teorías diferentes, inclusive empleadas complementariamente, acorde a la secuencia de eventos, la temporalidad del fenómeno y las preguntas de investigación (Thelen \& Mahoney, 2015, pp. 20-24).

\section{CONCLUSIÓN}

Este trabajo describió el desarrollo institucional del personal en el Chile post-autoritario (1990-2015) y determinó que este debe ser considerado como un caso de trayectoria dependiente, en el que el contexto político limitó la extensión de la incertidumbre y contingencia entre 1990 y 1994, sin que se produjera una coyuntura crítica que llevara a la interrupción abrupta de las reglas preexistentes.

Un conjunto de condiciones favoreció la restauración y actualización del modelo organizacional tradicional del personal en 1994. Al respecto, la influencia de los funcionarios dependió de su supervivencia bajo el autoritarismo, la que les confirió un recurso (i.e., experticia) que emplearon por algún tiempo después de 1990, sin poder vetar las reglas acordadas por el liderazgo parlamentario en 1994. En tal sentido, la clausura previa del parlamento no se tradujo en la supresión de su personal por la Junta ${ }^{13}$, por razones que solo se pueden conjeturar (i.e., redes con la élite política, neutralidad de su misión, transitoriedad del autoritarismo y/o necesidad de un parlamento futuro), lo que apuntaló a esta burocracia durante el autoritarismo.

Las reglas aprobadas en 1994 permitieron la reproducción de patrones de comportamiento ligados a las funciones del personal y generaron posteriormente una retroalimentación positiva para sus integrantes, quienes apoyaron el statu quo institucional. Asimismo, los cambios puntuales introducidos en ellas constituyeron manifestación de una "traducción", lo que fue posible por la resiliencia institucional de tales reglas, sin que implicaran un cambio sistémico al modelo organizacional citado y al funcionamiento del personal, por lo que la continuidad constituyó un rasgo característico del desarrollo institucional de aquel en el periodo. Presumiblemente, estas circunstancias hacen inviable una reversión del modelo organizacional citado en el corto plazo, pero no cierran la puerta a algún proceso de cambio institucional transformativo más adelante (e.g., deriva institucional).

\footnotetext{
${ }^{13}$ Esta supervivencia del personal existió también tras la clausura del parlamento en 1924 (Silva, 1997, pp. 75-76).
} 
Estos hallazgos sugieren comprender el desarrollo institucional -y el cambio institucional- como un proceso temporal que puede adoptar connotaciones incrementales o transformativas acordes al contexto, actores y ámbitos del sistema político, por lo que diferentes teorías, empleadas complementariamente inclusive, pueden explicar diversos aspectos de un fenómeno político, a partir de un diseño de investigación apropiado.

Por último, la información proporcionada por los entrevistados tuvo una importancia relativa en los resultados de la investigación. Dados los cargos desempeñados por ocho de ellos en el parlamento, su testimonio complementó pasajes oscuros de la documentación analizada y clarificó hechos de la última década del periodo. Por lo anterior, se puede estimar que existió complementariedad entre las fuentes de información, en relación con el objetivo de esta investigación.

\section{REFERENCIAS}

Aagard, K. (2017). The Evolution of a National Research Funding System: Transformative Change Through Layering and Displacement. Minerva 55, 279-297.

Ampuero, H. (2012). La modernización de la Cámara de Diputados, ¿tarea frustrada? Revista Chilena de Derecho y Ciencia Política 3(1), 115-130.

Baeza, J. (2012). Élites, path dependency y la falta de un pacto constitucional estable en Chile y Argentina: Un análisis comparativo-histórico. Política 50(2), 63-86.

Biblioteca del Congreso Nacional de Chile (s.f.). Historia de la Ley 19.297. Introduce Modificaciones a Ley $N^{\circ} 18.918$, Orgánica Constitucional del Congreso Nacional. Disponible en: https://www.bcn.cl/historiadelaley/nc/historia-de-la-ley/5733/ [consulta: 24 de agosto de 2018].

Bosch, G. (2018). The making of the German minimum wage: a case study of institutional change. Industrial Relations Journal 49(1), 19-33.

Brahm, M. (1997). Evaluación de experiencias de fortalecimiento de instituciones democráticas: El caso del Parlamento chileno. En Cámara de Diputados. Seminario y Encuentro Iberoamericano sobre Modernización y Reforma Parlamentaria, Tomo II (pp. 1-48). Valparaíso: Cámara de Diputados.

Busemeyer, M. \& Thelen, K. (2020). Institutional sources of business power. World Politics 72(3), 1-33.

Caballero, G. (2008). El cambio institucional de la economía española del franquismo a la democracia. Política y gobierno XV(2), 353-401.

Cámara de Diputados (1994). Sesiones de la Cámara de Diputados. Sesión 34a Ordinaria, martes 30 de agosto de 1994.

Cámara de Diputados (1996). Jornada de Trabajo. Cámara de Diputados. Algarrobo, Chile: Cámara de Diputados. 
Campbell, J. (2011). Institutional Reproduction and Change. En G. Morgan; J. Campbell; O. Pedersen, y R. Whitley (eds.). The Oxford Handbook of Comparative Institutional Analysis (pp. 87-115). New York: Oxford University Press.

Capoccia, G. (2015). Critical junctures and institutional change. En J. Mahoney \& K. Thelen (eds.). Advances in Comparative-Historical Analysis (pp. 147179). New York: Cambridge University Press.

Capoccia, G. (2016). When do Institutions "Bite". Historical Institutionalism and the Politics of Institutional Change. Comparative Political Studies 49(8), $1-33$.

Chagas, K. (2016). Dependencia da trajetória e mudança institucional nos processos de desenvolvimento. Revista Brasileira de Ciencia Política, 19, 253-275.

Conran, J. \& Thelen, K. (2016). Institutional Change. En O. Fioretos; T. Falleti y A. Sheingate (eds.). The Oxford Handbook of Historical Institutionalism (pp. 51-70). New York: Oxford University Press.

Djelic, M. (2010). Institutional Perspectives-Working Towards Coherence or Irreconcilable Diversity? En G. Morgan, J. Campbell, C. Crouch \& O. Pedersen (eds.). The Oxford Handbook of Comparative Institutional Analysis (pp. 1-40). New York: Oxford University Press.

Eckstein, H. (1993). Regarding Politics. Berkeley: University of California Press.

Feliú, X. (2004). La Biblioteca del Congreso Nacional entre 1960 y 1990: Procesos de cambio y continuidad. En D. Vásquez (ed.). 1883-2003: 120 años de la Biblioteca del Congreso Nacional (pp. 55-62). Santiago: La Biblioteca.

Fioretos, O., Falleti, T. \& Sheingate, A. (2016). Historical Institutionalism in Political Science. En O. Fioretos, T. Falleti \& A. Sheingate (eds.). The Oxford Handbook of Historical Institutionalism (pp. 4-28). New York: Oxford University Press.

Galvin, D. (2012). The transformation of political institutions: investments in institutional resources and gradual change in the national party committees. Studies in American Political Development 26(1), 50-70.

Gobierno de Chile (s.f.). Fortalecimiento de la Ética y Transparencia en el Congreso Nacional. Disponible en https://www.cl.undp.org/content/chile/es/ home/operations/projects/democratic_governance/etica_transparencia_ congreso_nacional.html [consulta: 14 de agosto de 2019].

Hall, P. (2011). Historical Institutionalism in Rationalist and Sociological Perspective. En J. Mahoney \& K. Thelen. Advances in Comparative-Historical Analysis (pp. 204-223). New York: Cambridge University Press.

Hall, P. (2016) Politics as a Process Structures in Space and Time. En O. Fioretos, T. Falleti \& A. Sheingate (eds.). The Oxford Handbook of Historical Institutionalism (pp. 31-50). New York: Oxford University Press.

Hoffmann, T. \& Loëwenberg, G. (2009). Path Dependence as an Explanation of the Institutional Stability of the German Parliament. German Politics 18(4), 469-484. 
Krasner, S. (1984). Approaches to the State. Alternative Conceptions and Historical Dynamics. Comparative Politics 16(2), 223-246.

Levi, M. (1997). A Model, a Method, and a Map: Rational Choice in Comparative and Historical Analysis. En M. Lichbach \& A. Zuckerman (eds.). Comparative Politics. Rationality, Culture, and Structure (pp. 19-41). New York: Cambridge University Press.

Lindegaard, L. (2013). Power and Change: Locating Institutional Change Theories in a Power Context. Disponible en: https://www.diis.dk/files/media/ publications/import/extra/wp2013-20_lili_power-change_web_1.pdf [consulta: 27 de septiembre de 2018].

Mahoney, J. (2001). The Legacies of Liberalism. Path Dependence and Political Regimes in Central America. Baltimore: The Johns Hopkins University Press.

Mahoney, J. \& Thelen, K. (2010). A Theory of Gradual Institutional Change. Explaining Institutional Change. Ambiguity, Agency, and Power (pp. 1-37). New York: Cambridge University Press.

Mahoney, J., Mohamedali, K. y Nguyen, C. (2016). Causality and time in historical institutionalism. En O. Fioretos, T. Falleti \& A. Sheingate (eds.). The Oxford Handbook of Historical Institutionalism (pp. 71-88). New York: Cambridge University Press.

North, D. (1990). Institutions, Institutional Change and Economic Performance. New York: Cambridge University Press.

Obando, I. (2011). El desarrollo del personal parlamentario chileno, 18341924. Universum 26(2), 187-213.

Obando, I. (2016). Análisis sobre la continuidad y cambio institucional del personal parlamentario bajo la Constitución Política de 1925. Ius et Praxis $22(2), 125-152$.

Oficina de Informaciones (1996). La Cámara de Diputados, situación administrativa, diagnóstico y eventuales soluciones. Valparaíso: Oficina de Informaciones.

Oficina de Informaciones de la Cámara de Diputados (2009). La Oficina de Informaciones de la Cámara de Diputados. 1990-2008. Documentos e Informes. Valparaíso: Oficina de Informaciones.

Palanza, V., Scartascini, C. \& Tommasi, M. (2013). A Tale of Two Latin American Congresses. Towards a Comparative Study of Institutionalization and Effectiveness. Buenos Aires: Universidad de San Andrés.

Pierson, P. (2004). Politics in Time. Princeton: Princeton University Press.

Pierson, P. (2015). Power and path dependence. En J. Mahoney \& K. Thelen (eds.). Advances in Comparative-Historical Analysis (pp. 123-146). New York: Cambridge University Press.

República de Chile (1989). Informa Proyecto de Ley Orgánica Constitucional sobre Congreso Nacional (Boletín $N^{\circ}$ 1152-06). Santiago: Honorable Junta de Gobierno, IV Comisión Legislativa.

Rosenblatt, F. y Toro, S. (2015). La arquitectura de la cooperación. Política y gobierno XXII(2), 255-281. 
Schickler, E. (2001). Disjointed Pluralism. Princeton: Princeton University Press.

Schickler, E. (2005). Institutional Development of Congress. En P. Quirk \& S. Binder (eds.). The Legislative Branch (pp. 35-62). New York: Oxford University Press.

Senado (1994). Diario de Sesiones del Senado. Sesión 27a Ordinaria, miércoles 31 de agosto de 1994. Disponible en www.senado.cl (consulta: 10 de enero de 2019).

Silva, A. (1997). Tratado de Derecho Constitucional, Vol. III. Santiago: Editorial Jurídica de Chile.

Sorensen, A. (2015). Taking path dependency seriously: an historical institutionalist research agenda in planning history. Disponible en: https://tandfonline-com.utalca.idm.oclc.org/doi/full/10.1080/02665433.2013.874299?scroll $=$ top $\&$ needAccess $=$ true [consulta: 28 de septiembre de 2018].

Soto, S. (2015). Congreso Nacional y Proceso Legislativo. Teoría y Práctica. Santiago: Thomson Reuters.

Streeck, W. \& Thelen, K. (2005). Introduction: Institutional Change in Advanced Political Economies. Beyond Continuity (pp. 1-39). New York: Cambridge University Press.

Tapia, J. (1960). La técnica legislativa. Santiago: Editorial Jurídica de Chile.

Thelen, K. (2003). How Institutions Evolve. En J. Mahoney \& D. Rueschmeyer (eds.). Comparative Historical Analysis in the Social Sciences (pp. 208-239). New York: Cambridge University Press.

Thelen, K. (2004). How Institutions Evolve. New York: Cambridge University Press.

Thelen, K. (2009). Institutional Change in Advanced Political Economies. Disponible en: https://onlinelibrary.wiley.com/doi/10.1111/j.1467-8543.2009. 00746.x [consulta: 24 de septiembre de 2018].

Thelen, K. (2014). Varieties of Liberalization and the New Politics of Social Solidarity. New York: Cambridge University Press.

Thelen. K. y Mahoney, J. (2015). Comparative historical analysis in contemporary political science. En J. Mahoney \& K. Thelen. Advances in Comparative-Historical Analysis (pp. 3-36). New York: Cambridge University Press.

Thelen, K. (2019). Transitions to the knowledge economy in Germany, Sweden and the Netherlands. Comparative Politics 51(2), 295-315.

Van der Heijden, J. (2011). Institutional layering: A review of the use of the concept. Politics 31(1), 9-18. 